\title{
Gastos hospitalares com crianças e adolescentes vítimas de violência, no Estado de Pernambuco, Brasil, em 1999
}

\author{
Hospital costs due to violence against \\ children and adolescents in Pernambuco \\ State, Brazil, during 1999
}

Roberto Natanael da Silva Mendonça ${ }^{1}$

João Guilherme Bezerra Alves 1

José Eulálio Cabral Filho 1

1 Instituto Materno
Infantil de Pernambuco.
Rua dos Coelhos 300,
Recife, PE 50070-550, Brasil.
joaoguilherme@imip.org.br

\begin{abstract}
This study focused on expenditures under the Brazilian National Unified Health System (SUS) due to hospitalization of children and adolescents as the result of violence in Pernambuco State in 1999. Data were collected from the Hospital Admittance Forms covering the State of Pernambuco. Some 9,220 hospital admissions due to external causes were identified, with a cost of $R \$ 3,659,558.62$. Mean cost of hospitalization for children and adolescents in Pernambuco State in 1999 was $R \$ 306.49$. Mean cost of hospitalization due to external causes was R\$396.91. Traumatic lesions were the most common reason for hospital admission (84.7\%). Violence against children and adolescents in Greater Metropolitan Recife accounted for $65.1 \%$ of hospital admissions and 77.9\% of all hospital costs in Pernambuco State.

Key words Violence; Hospital Costs; Wounds and Injuries; Child Health

Resumo Avaliaram-se os gastos do Sistema Único de Saúde (SUS) com internações hospitalares de crianças e adolescentes, vítimas de violência no Estado de Pernambuco, no ano de 1999. Utilizou-se o banco de dados do Sistema de Informações Hospitalares do DATASUS, construído a partir das informações contidas nas Autorizações de Internação Hospitalar (AIH). Foram registradas 9.220 internações por causas externas a um custo de $R \$ 3.659 .558,62$. O custo médio no Estado de Pernambuco nesse ano, para as internações por todos os diagnósticos na faixa etária de 0 a 19 anos, foi de $R \$ 306,49$. Quando se computaram apenas as causas externas, o custo médio passou para $R \$ 396,91$. Os traumatismos foram as principais causas desses internamentos $(84,7 \%)$. Na mesorregião Recife ocorreram 65,1\% das hospitalizações e 77,9\% dos gastos totais em todo o Estado.

Palavras-chave Violência; Custos Hospitalares; Ferimentos e Lesões; Saúde Infantil
\end{abstract}




\section{Introdução}

A violência é hoje um dos principais problemas de saúde pública tanto para os países ricos como para os pobres. Além de provocar forte impacto sobre as taxas de morbimortalidade, apresenta importantes repercussões econômicas. A violência custa anualmente US\$ 425 bilhões aos americanos (Farrel, 1993). Na América Latina, estima-se que os custos econômicos anuais de mortes e invalidez resultantes de atos violentos, representem aproximadamente $20 \%$ dos gastos totais de cada país com a saúde (Weaver \& Maddaleno, 1999).

É difícil avaliar por completo o verdadeiro impacto econômico da violência. Afora os custos médicos do tratamento e reabilitação da vítima, estão envolvidos gastos com os sistemas judiciário e penal, os custos sociais com a queda de produtividade, além de perdas materiais decorrentes dos agravos (Miller et al., 1993). Mesmo em países ricos, o aumento da demanda de serviços por vítimas da violência, ao lado da maior complexidade do atendimento ao paciente com trauma - muitas vezes envolvendo tecnologia complexa - está provocando a desativação de centros de trauma pela importante elevação dos custos. Os casos de violência chegam a consumir entre 8 a $10 \%$ do orçamento de cada hospital que mantém pronto-socorro (Clancy et al., 1994).

No Brasil, o fenômeno do incremento da violência vem sendo bem observado. Deslandes et al. (1998) detectaram um aumento de $69 \%$ nos gastos federais com o atendimento de vítimas de violência na Região Metropolitana do Rio de Janeiro, entre os anos de 1991 e 1993. Entretanto, apesar de elevados, ainda não são bem conhecidos os custos acarretados com os eventos violentos em nosso país. A obtenção desses dados econômicos, além de contribuir para a identificação do impacto da violência sobre a sociedade, apresenta um maior poder de sensibilização para as lideranças políticas do que as informações epidemiológicas. Serve ainda, como parâmetro para avaliação das intervenções postas em prática no combate à violência.

Considerando a importância do tema, bem como o fato de o estado de Pernambuco figurar com destaque no panorama da violência nacional, com importante crescimento da mortalidade por causas externas nos grupos etários mais jovens (Barros et al., 2001), foi objetivo deste estudo verificar os gastos do Sistema Único de Saúde (SUS) nesse estado com os internamentos de crianças e adolescentes por essas causas no ano de 1999.

\section{Metodologia}

Local do estudo

O Estado de Pernambuco possui cerca de 184 municípios, além do Distrito Estadual de Fernando de Noronha, sendo a capital do Estado a cidade de Recife - o município mais populoso, com cerca de 1.421.947 habitantes em agosto de 2000. Nesse ano, a população do estado era de 7.910.992 pessoas, com uma densidade demográfica de 78,37 habitantes $/ \mathrm{km}^{2}$ e uma concentração de $76,5 \%$ nas áreas urbanas. A população estimada para os grupos etários de 0 a 14 anos e de 15 a 19 correspondia, respectivamente, a $31,5 \%(2.497 .367)$ e 10,8\% (859.450) da população total. No ano de 1999, o total de leitos disponíveis no Estado para o SUS, em 303 hospitais, era de 21.916, incluindo as emergências, dos quais $11.906(54,33 \%)$ apenas na $\mathrm{Re}$ gião Metropolitana do Recife (CONDEPE, 2000).

\section{$\underline{\text { Dados }}$}

Foi realizado um estudo descritivo, cuja fonte de informação foram os arquivos reduzidos da base de dados, em CD-ROM, do Sistema de Informações Hospitalares do Sistema Único de Saúde (SIH-SUS), do Departamento de Informática do SUS (DATASUS), relativa ao ano de 1999 (MS, 1999) no Estado de Pernambuco, com informações hospitalares obtidas dos formulários de Autorização de Internação Hospitalar (AIH). Utilizou-se a AIH 1, que contém registro de identificação do paciente, procedimentos, diagnose e tratamento realizado, acompanhado dos respectivos códigos das afecções diagnosticadas. Foi construído um banco de dados com os códigos de diagnóstico principal, segundo a CID-10 (OMS, 1995), incluídos nos capítulos XIX e XX, sendo selecionada a população de 0 a 19 anos de idade.

\section{Resultados}

Durante o ano de 1999, ocorreram em Pernambuco 570.442 hospitalizações para todos os diagnósticos, sendo $190.985(33,4 \%)$ na faixa etária de 0 a 19 anos de idade. Destas, cerca de 9.220 foram motivadas por causas externas $(4,8 \%)$. Sua distribuição de freqüência por sexo e faixa etária encontra-se na Tabela 1.

O custo total dos internamentos para todas as faixas etárias em Pernambuco, no ano de 1999, representou para o SUS R\$212.073.716,90. Considerando-se apenas a faixa etária até os 19 anos, este valor foi de $\mathrm{R} \$ 58.382 .850,70$ (custo 
médio de $\mathrm{R} \$ 306,49$ por paciente). As afecções relativas aos capítulos XIX e XX, corresponderam a $6,27 \%$ desse total, com um custo médio de R \$ 396,91 por paciente.

Na Tabela 2, constata-se que os traumatismos foram a principal causa das hospitalizações por causas externas, no Estado, correspondendo isoladamente, a $84,7 \%$ de todos os casos. As queimaduras foram a segunda causa mais comum de internamento, predominando na faixa etária de 0 a 4 anos.

A Tabela 3 reproduz a distribuição do volume dos gastos em internamentos nas várias mesorregiões do Estado. A mesorregião Recife, isoladamente, consumiu cerca de $77,9 \%$ de todos os recursos despendidos em Pernambuco, em 1999, tendo sido os demais $22,1 \%$ distribuídos pelo conjunto das outras quatro mesorregiões.

Na distribuição por faixas etárias, observase que os maiores gastos nas mesorregiões do Recife, Agreste e São Francisco ocorreram na faixa etária dos 15-19 anos, o que representa $31,0 \%$ do valor total para 1999. Na mesorregião Recife, foram registrados $65,1 \%$ do total das internações no grupo etário de 0 a 19 anos, 13,7\% no sertão, $10,9 \%$ no agreste, $7,1 \%$ no Sertão do São Francisco e 3,2\% na Zona da Mata do Estado de Pernambuco.

Em relação aos custos por diagnóstico, observamos que os traumatismos responderam por $77,9 \%$ do total dos gastos, seguidos por queimaduras $15,2 \%$ e complicações e seqüelas de causas externas $5,2 \%$. Outras causas externas corresponderam a $1,7 \%$ dos gastos.

\section{Discussão}

No presente trabalho, buscou-se verificar os gastos do SUS com as internações por acidentes e violências, ou seja, foram descritos alguns dos denominados custos médicos diretos, já que não foram abordadas questões como o seguimento ambulatorial após alta e tratamentos de reabilitação. Esse custo está subestimado, haja vista aqueles que foram atendidos nas emergências e não hospitalizados, ou que procuraram e não conseguiram atendimento, ou ainda morreram no local da ocorrência, não entrando assim no cômputo geral do SUS.

Em Pernambuco, o custo das internações hospitalares por causas externas, no SUS, apresentaram um crescimento de $47,20 \%$ entre os anos de 1996 a 1999. O percentual gasto pelo SUS com todas as internações por causas externas no ano de 1999, correspondeu a algo em torno de $0,05 \%$ do Produto Interno Bruto do Estado (CONDEPE, 2000). Estudo pioneiro na es-
Tabela 1

Distribuição dos pacientes hospitalizados pelo SUS segundo sexo, por faixa etária de 0 a 19 anos. Pernambuco, Brasil, 1999.

\begin{tabular}{|c|c|c|c|c|c|}
\hline \multirow{3}{*}{$\begin{array}{l}\text { Faixa etária } \\
\text { (anos) }\end{array}$} & \multicolumn{4}{|c|}{ Gênero } & \multirow{3}{*}{$\begin{array}{c}\text { Total } \\
n\end{array}$} \\
\hline & \multicolumn{2}{|c|}{ Masculino } & \multicolumn{2}{|c|}{ Feminino } & \\
\hline & $n$ & $\%$ & $\mathrm{n}$ & $\%$ & \\
\hline $0-4$ & 1.185 & 59,0 & 823 & 41,0 & 2.008 \\
\hline $4-10$ & 1.621 & 68,4 & 747 & 31,6 & 2.368 \\
\hline $10-14$ & 1.892 & 77,6 & 544 & 22,4 & 2.436 \\
\hline $14-19$ & 1.891 & 78,5 & 517 & 21,5 & 2.408 \\
\hline Total & 6.589 & 71,4 & 2.631 & 28,6 & 9.220 \\
\hline
\end{tabular}

timativa de impacto econômico dos acidentes e violências no Brasil, revelou que as internações por estas causas em todo o país geraram um gasto anual que, em 1994, correspondia a $0,07 \%$ do produto interno bruto nacional (Iunes, 1997).

Lesões e envenenamentos consumiram, em 1994, cerca de $8 \%$ dos gastos totais do SUS com internações, quando considerado todo o país (Iunes, 1997). Em 1997, o gasto final com lesões correspondeu a $6,6 \%$ do total por todas as internações (Feijó \& Portela, 2001). Em Pernambuco, em 1999, essas afecções corresponderam a $6,27 \%$ do total, quando considerada a faixa etária de 0-19 anos.

O custo médio de internação por lesões e envenenamentos, em 1999, foi de R\$ 511,26 no Brasil, sendo de R\$ 471,95 em Pernambuco (DATASUS, 2000), porém entre crianças e adolescentes o valor médio foi de R\$396,91 em nosso Estado, com variações de $\mathrm{R} \$ 307,29$ no mês de março a $\mathrm{R} \$ 533,17$, em novembro. Para que se possa estabelecer uma comparação, em 1994 essas hospitalizações custaram, em média, $\mathrm{R} \$ 328,78$, quando consideradas todas as faixas etárias no Brasil (Iunes, 1997). Dados do Ministério da Saúde demonstram que o valor médio dessas internações, no ano de 1997, para todas as idades, foi de $\mathrm{R} \$ 400,66$ (DATASUS, 2000).

Avaliando-se as internações na faixa etária de 0 a 19 anos, observa-se que os 9.220 casos existentes corresponderam a $35 \%$ do total de internações por acidentes e violências, para todas as idades, em Pernambuco, no ano de 1999. Isso representa uma taxa de internação de 2,7 / 1.000 habitantes, na faixa etária considerada.

No Brasil, esses números podem não ser tão elevados como nos outros países pelo fato de que aqui eles se referem apenas às internações pagas pelo SUS, não estando computados os casos atendidos em hospitais não conveniados. Acresce ainda, que os atendimentos de pronto- 
Tabela 2

Distribuição dos diagnósticos de internamento, na faixa etária de 0 a 19 anos. Pernambuco, Brasil, 1999.

\begin{tabular}{|c|c|c|c|c|c|c|c|c|c|c|}
\hline \multirow[t]{3}{*}{ Diagnóstico } & \multicolumn{8}{|c|}{ Faixa etária } & \multicolumn{2}{|c|}{ Total } \\
\hline & \multicolumn{2}{|c|}{$0-4$} & \multicolumn{2}{|c|}{$5-9$} & \multicolumn{2}{|c|}{$10-14$} & \multicolumn{2}{|c|}{$15-19$} & & \\
\hline & $\mathrm{n}$ & $\%$ & $\mathrm{n}$ & $\%$ & $\mathrm{n}$ & $\%$ & $\mathrm{n}$ & $\%$ & $\mathrm{n}$ & $\%$ \\
\hline Traumatismos & 1.396 & 69,6 & 2.099 & 88,6 & 2.239 & 91,8 & 2.073 & 86,1 & 7.807 & 84,7 \\
\hline Queimaduras & 345 & 17,2 & 110 & 4,6 & 47 & 1,9 & 40 & 1,7 & 542 & 5,9 \\
\hline $\begin{array}{l}\text { Complicações e seqüelas } \\
\text { de causas externas }\end{array}$ & 65 & 3,2 & 68 & 2,9 & 92 & 3,8 & 193 & 8,0 & 418 & 4,5 \\
\hline Efeitos tóxicos & 93 & 4,6 & 48 & 2,0 & 27 & 1,1 & 64 & 2,7 & 232 & 2,5 \\
\hline Intoxicações & 70 & 3,5 & 25 & 1,1 & 20 & 0,8 & 34 & 1,4 & 149 & 1,6 \\
\hline Corpo estranho & 27 & 1,3 & 12 & 0,5 & 7 & 0,3 & 2 & 0,1 & 48 & 0,5 \\
\hline Outras causas externas & 12 & 0,6 & 6 & 0,3 & 4 & 0,2 & 2 & 0,1 & 24 & 0,3 \\
\hline Total & 2.008 & 100,0 & 2.368 & 100,0 & 2.436 & 100,0 & 2.408 & 100,0 & 9.220 & 100,0 \\
\hline
\end{tabular}

Tabela 3

Distribuição do volume dos gastos em internamentos de crianças e adolescentes nas várias mesorregiões do Estado de Pernambuco, Brasil, 1999.

\begin{tabular}{|c|c|c|c|c|c|c|}
\hline \multirow[t]{2}{*}{ Mesorregião } & \multicolumn{4}{|c|}{ Faixa etária } & \multicolumn{2}{|c|}{ Gastos totais } \\
\hline & $0-4$ & $5-9$ & $10-14$ & $15-19$ & $\mathrm{R} \$$ & $\%$ \\
\hline Recife & $676.060,22$ & $582.434,29$ & $679.499,77$ & $911.280,74$ & $2.849 .275,02$ & 77,86 \\
\hline Sertão & $72.627,76$ & $95.466,51$ & $90.217,07$ & $76.120,83$ & $334.432,17$ & 9,14 \\
\hline Agreste & $43.973,52$ & $72.161,35$ & $61.561,70$ & $89.620,37$ & $267.316,94$ & 7,30 \\
\hline São Francisco & $30.490,74$ & $35.403,78$ & $29.856,12$ & $42.669,51$ & $138.420,15$ & 3,78 \\
\hline Mata & $17.631,36$ & $13.851,70$ & $20.776,37$ & $17.854,91$ & $70.114,34$ & 1,92 \\
\hline Total & $840.783,60$ & $799.317,63$ & $881.911,03$ & $1.137 .546,36$ & $3.659 .558,62$ & 100,00 \\
\hline
\end{tabular}

socorro não são computados através das AIHs e entretanto, é nesse tipo de serviço que muitos dos casos de lesões e envenenamentos são atendidos e resolvidos.

Quando avaliados isoladamente, os traumatismos foram as principais causas de internamento para todas as faixas etárias, perfazendo $84,7 \%$ e com maiores freqüências na faixa de 5-19 anos. A distribuição de freqüência dos internamentos por mesorregiões do Estado, revelou uma concentração das hospitalizações por acidentes e violência na mesorregião Recife. Quando se avalia os gastos realizados nos internamentos na cidade do Recife, verifica-se que $91,9 \%$ dos recursos financeiros utilizados na mesorregião Recife foram na capital do Estado. Isso provavelmente decorre não apenas em virtude de uma maior freqüência de acidentes e violências no Recife, mas também, devido à remoção dos casos mais graves das cida- des onde se verificaram os danos para os hospitais de referência, na capital. Isso reforça o conceito de que a cidade do Recife e sua região metropolitana, são os principais responsáveis pela violência que ocorre no Estado e respondem por um dos mais expressivos índices de violência no país (Barros et al., 2001; Lima \& Ximenes, 1998). Em todo o Brasil, a concentração dos acidentes e violências é visivelmente mais clara nas áreas urbanas, que acumulam $75 \%$ do total das mortes por causas violentas (DATASUS, 2000; Freitas et al., 2000). Em áreas urbanas, inclusive, já se observou que o risco de recorrência do trauma é elevado, podendo chegar até a 44\% (Cruz, 1999; Poole, 1993).

Do exposto, resulta que a mensuração dos gastos para o sistema público de saúde gerado por todas as formas de violência, ainda não pode ser realizada em toda a sua magnitude, já que a avaliação dos custos indiretos carece de 
dados fidedignos que a fundamentem, e a própria medição dos custos diretos não médicos é difícil de ser realizada. Dessa forma, para uma completa avaliação do impacto da violência sobre a saúde pública e as suas repercussões sócio-econômicas, é fundamental que pesquisas com esse intuito sejam continuamente fomentadas.

Para que políticas públicas de combate à violência sejam eficazes, faz-se necessário, em paralelo às medidas de segurança, que a "saúde pública” também estabeleça quais as populações de risco em cada região e quais as características das lesões sofridas por essa mesma população. Isto servirá como subsídio às autoridades, para que possam estabelecer as priorida-

\section{Referências}

BARROS, M. D. A.; XIMENES, R. \& LIMA, M. L. C., 2001. Mortalidade por causas externas em crianças e adolescentes: Tendências de 1979 a 1995. Revista de Saúde Pública, 35:142-149.

CLANCY, T. V.; MISICK, L. N.; COVINGTON, D.; CHURCHILL, M. P. \& MAXWELL, J. G., 1994. The financial impact of intentional violence on community hospitals. Journal of Trauma, 37:1-4.

CONDEPE (Instituto de Planejamento de Pernambuco), 2000. Pernambuco em Dados - 2000. Recife: CONDEPE.

CRUZ, J. M., 1999. El impacto psicosocial de la violencia en San Salvador. Revista Panamericana de Salud Pública, 5:295-302.

DATASUS (Departamento de Informática do SUS), 2000. Morbidade Hospitalar do SUS - CID 10. 29 Maio 2001 <http://www.datasus.gov.br/cgi/defto htm.exe?sih/mbr.def>

DESLANDES, S. F.; SILVA, C. M. F. P. \& UGÁ, M. A. D., 1998. O custo do atendimento emergencial às vítimas de violências em dois hospitais do Rio de Janeiro. Cadernos de Saúde Pública, 14:287-299.

FARREL, C., 1993. The economics of crime. Business Week, 193:72-80.

FEIJÓ, M. C. C. \& PORTELA, M. C., 2001. Variação no custo de internações hospitalares por lesões: Os casos dos traumatismos cranianos e acidentes por armas de fogo. Cadernos de Saúde Pública, 17:627-637.

FREITAS, E. D.; PAIM, J. S.; SILVA, L. M. \& COSTA, M. D., 2000. Evolução e distribuição espacial da mortalidade por causas externas em Salvador, Bahia, Brasil. Cadernos de Saúde Pública, 16:1059-1070. des peculiares de cada bairro, cidade ou estado.

Os custos diretos médicos, conforme descritos neste trabalho, são elevados e estariam muito melhor empregados se, porventura, pudessem ser aplicados em educação e saúde preventiva e não no tratamento, muitas das vezes paliativo, de lesões que, em níveis crescentes, têm sido intencionalmente infligidas. Os custos financeiros para o país têm sido muito elevados, como os têm sido os custos sociais. Urge a busca de soluções em caráter definitivo para este grave problema, que, embora não tenha solução a curto prazo, pode ser combatido em várias frentes e cujos resultados far-se-ão sentir a médio e longo prazos.
IUNES, R. F., 1997. Impacto econômico das causas externas no Brasil: Esforço de mensuração. Revista de Saúde Pública, 31:38-46.

LIMA, M. C. L. \& XIMENES, R., 1998. Violência e morte: Diferenciais da mortalidade por causas externas no espaço urbano do Recife, 1991. $\mathrm{Ca}$ dernos de Saúde Pública, 14:829-840.

MILLER, T. R.; COHEN, M. A. \& ROSSMAN, S. B., 1993. Victim costs of violence crime and resulting injuries. Health Affairs, 12:195-197.

MS (Ministério da Saúde), 1999. Movimento de Autorização de Internação Hospitalar. Arquivos Reduzidos, 1999. Brasília: MS.

OMS (Organização Mundial da Saúde), 1995. Classificação Estatística Internacional de Doenças e Problemas Relacionados à Saúde, 10a Revisão. São Paulo: Centro Colaborador da OMS para a Classificação de Doenças em Português.

POOLE, G. V., 1993. Trauma is a recurrent disease. Surgery, 113:608-611.

WEAVER, K. \& MADDALENO, M., 1999. Youth violence in Latin America: Current situation and violence prevention strategies. Revista Panamericana de Salud Pública, 5:338-343.

Recebido em 20 de julho de 2001

Versão final reapresentada em 22 de fevereiro de 2002 Aprovado em 1 de abril de 2002 